\title{
AXISYMMETRIC ELECTRIC FIELD CALCULATION WITH ZONAL HARMONIC EXPANSION
}

\section{F. Glück ${ }^{1,2, *}$}

${ }^{1}$ Karlsruhe Institute of Technology, IEKP, POB 3640, 76021 Karlsruhe, Germany

${ }^{2}$ KFKI, RMKI, H-1525 Budapest, POB 49, Hungary

Abstract - The electric potential and field of an axially symmetric electric system can be computed by expansion of the central and remote zonal harmonics, using the Legendre polynomials. Garrett showed the usefulness of the zonal harmonic expansion for magnetic field calculations, and the similar radial series expansion has been widely used in electron optics. In this paper, we summarize our experience of using the zonal harmonic expansion for practically interesting axisymmetric electric field computations. This method provides very accurate potential and field values, and it is much faster than calculations with elliptic integrals. We present formulas for the central and remote expansions and for the coefficients of the zonal harmonics (source constants) in the case of general axisymmetric electrodes and dielectrics. We also discuss the general convergence properties of the zonal harmonic series (proof, rate of convergence, and connection with complex series). Practical considerations about the computation method are given at the end. In our appendix, one can find many useful formulas about properties of the Legendre polynomials, various derivatives of the zonal harmonic functions, and a simple numerical integration algorithm.

\section{INTRODUCTION}

Electric field calculation is important in many areas of physics: electron and ion optics, charged particle beams, charged particle traps, electron microscopy, electron spectroscopy, plasma and ion sources, electron guns, etc. [1-3]. A special kind of electron and ion energy spectroscopy is realized by the MAC-E filter spectrometers, where integral

Received 21 April 2011, Accepted 9 July 2011, Scheduled 23 July 2011

* Corresponding author: Ferenc Glück (ferenc.glueck@kit.edu). 
energy spectrum is measured by the combination of electrostatic retardation and magnetic adiabatic collimation. Examples are the Mainz and Troitsk electron spectrometers $[4,5]$, the aSPECT proton spectrometer $[6,7]$, the WITCH ion spectrometer $[8,9]$, and the KATRIN pre- and main electron spectrometers [10].

Various numerical methods exist for electric field computations $[2,3,11]$ : the finite difference method (FDM), the finite element method (FEM), and the boundary element method (BEM). Field computation with high accuracy in FDM and FEM is rather difficult. With BEM, however, the situation is much better. Although the calculated charge density distribution with BEM has some numerical error (it deviates somewhat from the real charge density), with a fixed charge density distribution it is possible to compute the potential and field at any arbitrary point with extremely high accuracy. This is an important advantage of BEM against FDM and FEM. Unfortunately, the Coulomb integration with summation over the many subelements of the discretized electrode surface is rather slow, even with axisymmetric electrodes (using elliptic integrals). It is expedient to replace the slow Coulomb integration-summation of BEM with a faster computation method, which at the same time keeps the high accuracy of the Coulomb integration.

It has been known for a long time [12-16] that axisymmetric electric and magnetic fields can be calculated by zonal harmonic expansion. Garrett showed in several papers [17-20] that in the case of axisymmetric magnetic systems the zonal harmonic expansion method has several practical advantages relative to the more widely known elliptic integral method. The main advantage is the speed: the zonal harmonic method is in some cases 100 or even 1000 times faster than the computation with the elliptic integrals. In the case of axisymmetric electric systems it has been widely known that the off-axis electric field and potential within some region not far from the symmetry axis can be expressed with the on-axis potential function (or with the higher derivatives of the on-axis potential), and the corresponding radial series expansion formulas can be found in many electron optics books [1-3]. Nevertheless, to our knowledge, before 2002 the zonal harmonic expansion method has not been used for practical electric field calculations.

The electric potential of an arbitrary electric system in a sourcefree region (vacuum) can be generally written as an expansion of the spherical harmonics, which are proportional to the associated Legendre polynomials $P_{n}^{m}(\cos \theta)[12,14-16]$. In the special case of axially symmetric electric systems the absence of the azimuthal dependence reduces the problem to the simpler zonal harmonic expansion. Defining 
an arbitrary reference point on the symmetry axis (we shall call it a source point), the central and remote solid zonal harmonics are the functions $\rho^{n} P_{n}(\cos \theta)$ and $\rho^{-(n+1)} P_{n}(\cos \theta)$, where $\rho$ is the distance between the source point and the field point, $\theta$ denotes the angle between the symmetry axis and the line connecting the source and field points, and $P_{n}(\cos \theta)$ is the Legendre polynomial of order $n$. Within a spherical region inside the electrodes (central region), with the source point as the center of the sphere, the electric potential can be expanded in central zonal harmonics; the radius of the sphere ( $\rho_{\text {cen }}$ : central convergence radius) is the minimal distance between the source point and the electrodes (see Fig. 1 in Section 2). Defining the remote convergence radius $\rho_{\text {rem }}$ by the maximal distance between the source point and the electrodes, within the remote region outside the electrodes (at field points $\rho>\rho_{\text {rem }}$ ) the potential can be expanded in remote zonal harmonics. The expansion formulas for the potential and the field can be found in Section 2. These central and remote expansion series are convergent only for $\rho<\rho_{\text {cen }}$ and $\rho>\rho_{\text {rem }}$, respectively. Let us define the convergence ratio $\mathcal{R}_{c}=\rho / \rho_{\text {cen }}$ for the central expansion and $\mathcal{R}_{c}=\rho_{\text {rem }} / \rho$ for the remote one. The rate of convergence is fast if the convergence ratio is small, and slow if $\mathcal{R}_{c}$ is close to 1 ; for $\mathcal{R}_{c}>1$ the series are divergent. Section 6 contains a mathematical proof of the convergence of the zonal harmonic expansions, and examples for the rate of convergence as function of the convergence ratio. In addition, we show there also the connection between the convergence of zonal harmonic expansions and complex power series.

In order to use the zonal harmonic expansion formulas (Eqs. (2)(4), (9)-(11) in Section 2) for the potential and field calculations, we have to know the coefficients $\Phi_{n}^{c e n}$ and $\Phi_{n}^{r e m}$ in these expressions; following the terminology of Garrett, we call these coefficients source constants. Section 3 contains formulas of source constants for a charged ring, and in Sections 4 and 5 one can find expressions of source constants for general axisymmetric electrodes and dielectrics. Note that all the field expansion and source constant formulas in our paper have been tested by comparisons with our computer codes.

The source constants depend on the source point and on the electric system properties: geometry of the electrodes and dielectrics, potential of the electrodes and permittivity of the dielectrics. In order to use our formulas for the source constant evaluations, one has to compute first the surface and volume charge density distributions of the electrodes and dielectrics. In the case of the boundary element method, this is no problem at all: the charge density calculation is a necessary step during the field computation process. With the knowledge of the charge density, the potential and field can also be 
computed by elliptic integrals, but this needs a lot of computation time (as we have already mentioned above); the zonal harmonic method is much faster. In the FDM and FEM, the charge density is usually not computed. Nevertheless, it is possible to derive it from the field: the charge density $\sigma$ on the metallic electrode surface can be calculated by the formula $\sigma=\varepsilon_{0} E$ (in SI units), where $E$ denotes the electric field on the electrode surface. One could of course argue: why take an additional step in FDM or FEM to calculate the field (far from the electrodes) by the zonal harmonic expansion, if it is already known from the usual FDM or FEM procedure? The answer is the following: for a fixed charge density distribution the zonal harmonic method is able to provide a potential or field distribution with an extremely high accuracy (for example: close to double precision), which could be advantageous, for example, for trajectory calculations that might need this high accuracy. One has to emphasize the term 'for fixed charge density' in the above sentence: it is usually not possible to compute very accurately the charge density distribution (even a single precision calculation could be quite difficult). Therefore, the calculated potential or field has an error due to the charge density error; the high accuracy of the computed field by the zonal harmonic method is relative to the charge density distribution.

The zonal harmonic expansion method is very fast and accurate, and these features make it ideal for high precision trajectory computations. Using this method, the electric field can be computed during the particle tracking 'on-line', i.e., no two-dimensional interpolation grid has to be calculated prior to the tracking. Instead, only the one-dimensional source point grid, containing the source constants at the source points, has to be computed in advance. If one insists on using the interpolation method for tracking simulations, then the computation of the interpolation grid is much faster with the zonal harmonic method than with the elliptic integrals.

Based on the publications of Garrett [17-20], we developed the zonal harmonic expansion method for axisymmetric electric field calculations. Using this method, together with the boundary element method for charge density calculation, we have written several FORTRAN and C codes for potential and field calculations of axially symmetric electrodes. These codes have been used for electromagnetic design studies and/or trajectory calculations connected with the aSPECT proton spectrometer [6,7], the Mainz neutrino mass spectrometer $[4,21]$, the WITCH ion spectrometer $[8,9]$, the Nab neutron decay spectrometer [22], and various axisymmetric electrode systems of the KATRIN experiment [10,23-32]. Note that all the diploma theses and dissertations cited in our paper can be 
found either on the KATRIN homepage [33] or on the working group homepage of Weinheimer [34]. Based upon our C codes, further electric field simulation $\mathrm{C}$ and $\mathrm{C}++$ codes have been written by various students at the University of Münster [35], at MIT [36], and at KIT [37]. The zonal harmonic method presented in this paper has been included into the $\mathrm{C}++$ simulation package KASSIOPEIA of the KATRIN experiment [38].

\section{ZONAL HARMONIC EXPANSION FOR THE ELECTRIC POTENTIAL AND FIELD}

Let us assume that we have an axially symmetric electric system, the axis $z$ being the symmetry axis. Fig. 1 shows a simple electrode system with 2 electrodes (E1 and E2). Let us define an arbitrary reference point $S\left(z_{0}, 0\right)$ on the symmetry axis: we shall call it a source point. An arbitrary space point, where we want to calculate the electric potential and field, will be called a field point; it has the Descartes coordinates $x, y, z$. This field point (denoted by $F$ in Fig. 1) can be defined by the cylindrical coordinates $z$ and $r$ (where $r=\sqrt{x^{2}+y^{2}}$ ), or by the distance $\rho$ between the source point and the field point, and by the angle $\theta$ between the symmetry axis $z$ and the line connecting these 2 points (due to the axial symmetry, the azimuthal angle of the field point around the symmetry axis is not relevant). We shall use quite

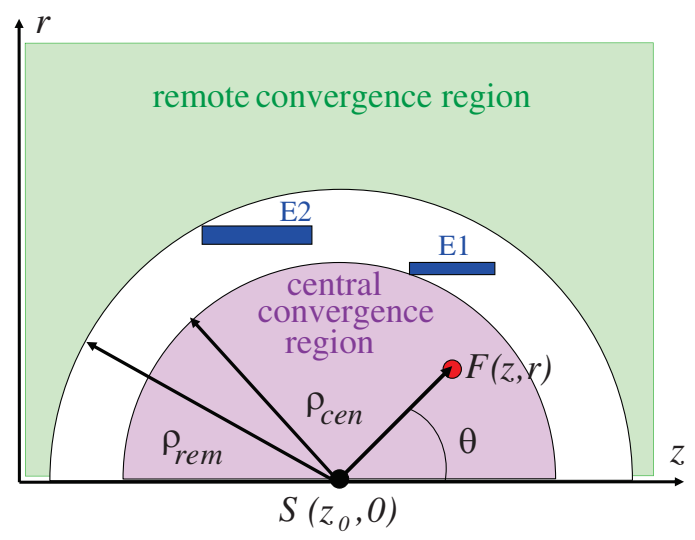

Figure 1. Electrodes E1 and E2, with field point $F$ and source point $S$, and with the central $\left(\rho<\rho_{c e n}\right)$ and remote $\left(\rho>\rho_{\text {rem }}\right)$ convergence regions. 
often the parameters $\rho, u$ and $s$ :

$$
\rho=\sqrt{\left(z-z_{0}\right)^{2}+r^{2}}, u=\cos \theta=\left(z-z_{0}\right) / \rho, s=\sin \theta=\sqrt{1-u^{2}}=r / \rho .
$$

We assume that the electric system is constrained inside a spherical shell, with the source point $S\left(z_{0}, 0\right)$ as its center. There are no electrodes or dielectrics inside the sphere with center $S$ and radius $\rho_{\text {cen }}$, and outside the sphere with center $S$ and radius $\rho_{\text {rem }}$. We call the area $\rho<\rho_{\text {cen }}$ central region and the area $\rho>\rho_{\text {rem }}$ remote region (see Fig. 1). The central convergence radius $\rho_{\text {cen }}$ is the minimal distance of the source point $S$ from the electrodes and dielectrics. Similarly, the remote convergence radius $\rho_{\text {rem }}$ is the maximal distance of the source point from the electrodes and dielectrics.

The central and remote regions are free from electric charges, which are the sources of the electric field. Therefore, the electric potential in these regions can be written as an expansion of the central zonal harmonics $\rho^{n} P_{n}(u)$ and of the remote zonal harmonics $\rho^{-(n+1)} P_{n}(u)$, which satisfy the Laplace equation $\left(P_{n}(u)\right.$ is the Legendre polynomial of order $n$ ).

\subsection{Central Region}

In the central region, we get the following expansion formulas for the electric potential $\Phi$ and the axial and radial electric field components $E_{z}$ and $E_{r}$ :

$$
\begin{aligned}
\Phi(z, r) & =\sum_{n=0}^{\infty} \Phi_{n}^{c e n}\left(\frac{\rho}{\rho_{c e n}}\right)^{n} P_{n}(u), \\
E_{z}(z, r) & =-\frac{1}{\rho_{c e n}} \sum_{n=0}^{\infty} \Phi_{n+1}^{c e n} \cdot(n+1)\left(\frac{\rho}{\rho_{c e n}}\right)^{n} P_{n}(u), \\
E_{r}(z, r) & =\frac{s}{\rho_{c e n}} \sum_{n=0}^{\infty} \Phi_{n+1}^{c e n}\left(\frac{\rho}{\rho_{c e n}}\right)^{n} P_{n}^{\prime}(u) .
\end{aligned}
$$

Here $P_{n}^{\prime}(u)=d P_{n}(u) / d u$ denotes the first derivative of the Legendre polynomial of order $n$. In order to compute the $P_{n}(u)$ and $P_{n}^{\prime}(u)$ values for very high indices $n$, one can use the recurrence relations (A11) and (A12) of Appendix A. Using Eqs. (A20)-(A28) and (B1)-(B8) in Appendices A and B, we have verified that the above formulas satisfy the fundamental static electric field equations in vacuum:

$$
\Delta \Phi=0, \quad \nabla \cdot \mathbf{E}=0, \quad \nabla \times \mathbf{E}=0, \quad \mathbf{E}=-\nabla \Phi .
$$

We have defined the coefficients $\Phi_{n}^{\text {cen }}(n=0,1, \ldots)$ so that each of them has the dimension of the electric potential $\Phi$, and $\Phi_{0}^{c e n}$ is 
equal to the potential at the source point $S\left(z_{0}, 0\right)$. We shall call these coefficients central source constants: they represent the electric field sources (electric charges and dipoles) inside the central region. They depend on the electric sources (electric system geometry, electrode potentials, dielectric permittivities), and on the given source point: $\Phi_{n}^{c e n}=\Phi_{n}^{c e n}\left(z_{0}\right)$. In the following 3 sections, we present source constant formulas for various kinds of electric systems. Here, we show some general properties of the source constants. First, the central source constants $\Phi_{n}^{c e n}$ are proportional to the higher derivatives of the onaxis potential function $\Phi_{0}(z)$ at the source point $S\left(z_{0}, 0\right)$. In order to understand this relation, let us take a special field point on the symmetry axis $(r=0)$ with $z>z_{0}$. Then $\theta=0, u=\cos \theta=1$, $P_{n}(1)=1$ (see Eq. (A4) in Appendix A), $\rho=z-z_{0}$, therefore from Eq. (2) we get

$$
\Phi(z, 0)=\Phi_{0}(z)=\sum_{n=0}^{\infty} \Phi_{n}^{c e n} \frac{1}{\rho_{c e n}^{n}}\left(z-z_{0}\right)^{n} .
$$

Comparing this equation with the general Taylor expansion formula of the on-axis potential $\Phi_{0}(z)$ around the source point $z_{0}$

$$
\Phi_{0}(z)=\sum_{n=0}^{\infty} \frac{1}{n !} \Phi_{0}^{(n)}\left(z_{0}\right)\left(z-z_{0}\right)^{n}, \quad \Phi_{0}^{(n)}\left(z_{0}\right)=\frac{d^{n} \Phi_{0}}{d z^{n}}\left(z_{0}\right),
$$

we get the relation:

$$
\Phi_{n}^{c e n}=\Phi_{n}^{c e n}\left(z_{0}\right)=\frac{\rho_{c e n}^{n}}{n !} \Phi_{0}^{(n)}\left(z_{0}\right) .
$$

The central zonal harmonic expansions in Eqs. (2)-(4) are convergent only for $\rho<\rho_{c e n}$. The convergence is fast if the convergence ratio $\mathcal{R}_{c}=\rho / \rho_{\text {cen }}$ is small, and rather slow if $\rho$ is close to $\rho_{c e n}\left(\mathcal{R}_{c}\right.$ is close to 1 ) (in this case a large number of terms have to be evaluated to get a prescribed accuracy). For $\rho>\rho_{c e n}\left(\mathcal{R}_{c}>1\right)$ the above expansions should not be used, because they provide then meaningless results, due to their divergence. Various considerations about the convergence properties of these formulas can be found in Section 6 .

\subsection{Radial Series Expansion}

It is well known in electron optics that for an axially symmetric electrode system the off-axis electric potential and field not too far from the symmetry axis are completely determined by the on-axis potential. The off-axis potential can be expressed by the radial series expansion that contains the higher derivatives of the on-axis potential [1-3]. 
The radial series expansion is a special case of the more general central zonal harmonic expansion. Namely, in the case of the radial series expansion the field point and the source point have the same axial coordinate values: $z=z_{0}$. That means: $\theta=90^{\circ}$ (the line connecting the field and source points is perpendicular to the $z$ axis), $u=0$ and $\rho=r$. Using Eq. (A5) in Appendix A and the connection between the central source constants and the higher derivatives $\Phi_{0}^{(n)}(z)$ in Eq. (7), we obtain

$$
\Phi(z, r)=\sum_{n=0}^{\infty} \frac{(-1)^{n}}{\left(2^{n} n !\right)^{2}} \Phi_{0}^{(2 n)}(z) r^{2 n} .
$$

This is the radial series expansion for the electric potential, which is presented in most electron optics books [1-3]. Similarly to the central zonal harmonic expansion, Eq. (8) is convergent only for $r<\rho_{c e n}$.

With the knowledge of the higher derivatives or of the central source constants at the axial point $z_{0}$, the radial series expansion makes possible the calculation of the potential and field at points of the 2dimensional plane $z=z_{0}$ (which is perpendicular to the axis $z$ ). On the other hand, using the zonal harmonic expansion, the field calculation is possible within a 3 -dimensional region (the sphere $\rho<\rho_{\text {cen }}$ ). Changing the coordinate $z$ of the field point, one needs different source constants for the radial series expansion, since for this calculation method the field and source points should have the same axial coordinate values. In the case of the zonal harmonic expansion, this complication is not present: one can use the same central source constants for all field points which are inside the convergence sphere with radius $\rho_{c e n}$ and center $\left(z_{0}, 0\right)$.

To use the radial series method for charged particle tracking, one has to compute the source constants for a rather dense source point distribution, and one has to interpolate the field between two neighboring source points. The zonal harmonic expansion method is free from these complications; the distance between two neighboring source points can be rather large (but a few times smaller than $\rho_{c e n}$ ), and no field interpolation is necessary.

\subsection{Remote Region}

In the case of field points with $\rho>\rho_{\text {rem }}$ (remote region) the electric potential can be expressed as an expansion of the remote zonal harmonic functions $\rho^{-(n+1)} P_{n}(u)$. We get the following remote zonal 
harmonic expansion formulas:

$$
\begin{aligned}
\Phi(z, r) & =\sum_{n=0}^{\infty} \Phi_{n}^{r e m}\left(\frac{\rho_{\text {rem }}}{\rho}\right)^{n+1} P_{n}(u), \\
E_{z}(z, r) & =\frac{1}{\rho_{\text {rem }}} \sum_{n=1}^{\infty} \Phi_{n-1}^{r e m} \cdot n\left(\frac{\rho_{\text {rem }}}{\rho}\right)^{n+1} P_{n}(u), \\
E_{r}(z, r) & =\frac{s}{\rho_{\text {rem }}} \sum_{n=1}^{\infty} \Phi_{n-1}^{r e m}\left(\frac{\rho_{\text {rem }}}{\rho}\right)^{n+1} P_{n}^{\prime}(u) .
\end{aligned}
$$

Using Eqs. (A20)-(A28) and (B1)-(B8) in Appendices A and B, we have verified that the above formulas satisfy the fundamental static electric field equations in vacuum: $\Delta \Phi=0, \nabla \cdot \mathbf{E}=0, \nabla \times \mathbf{E}=0$, $\mathbf{E}=-\nabla \Phi$.

The coefficients $\Phi_{n}^{r e m}(n=0,1, \ldots)$ are the remote source constants: they represent the electric field sources (charges) in the remote region. They have the dimension of the electric potential, and they depend on the electric sources and on the given source point: $\Phi_{n}^{r e m}=\Phi_{n}^{r e m}\left(z_{0}\right)$. In the following 3 sections, we present remote source constant formulas for various kinds of electric systems (with derivations).

The remote zonal harmonic expansions correspond to the multipole expansion of the electric potential and field, for axisymmetric systems. The first term in each expansion corresponds to the charge, the second to dipole, the third to quadrupole, etc. (see Refs. $[13,14,39,40])$. The remote source constants $\Phi_{n}^{\text {rem }}$ are proportional to the axisymmetric multipole electric moments. For example

$$
\Phi_{0}^{r e m}=\frac{1}{4 \pi \varepsilon_{0}} \frac{Q}{\rho_{\text {rem }}}, \quad \Phi_{1}^{r e m}=\frac{1}{4 \pi \varepsilon_{0}} \frac{p_{z}}{\rho_{\text {rem }}^{2}},
$$

where $Q$ is the electric charge, and $p_{z}$ denotes the electric dipole moment of the system (in case of axial symmetry only the axial component is non-zero). Substituting these expressions into Eqs. (9)(11), we obtain the point charge and dipole formulas for the electric potential and field. Note that we use SI units throughout our paper.

The above remote zonal harmonic expansion formulas are convergent only for $\rho>\rho_{\text {rem }}$. The convergence is fast if the convergence ratio $\mathcal{R}_{c}=\rho_{\text {rem }} / \rho$ is small, and rather slow if $\rho$ is close to $\rho_{\text {rem }}\left(\mathcal{R}_{c}\right.$ is close to 1$)$; in this case a large number of terms have to be evaluated, in order to get a prescribed accuracy. For $\rho<\rho_{\text {rem }}\left(\mathcal{R}_{c}>1\right)$ the above expansions should not be used, because they provide then meaningless results (due to their divergence). Various considerations 
about the convergence properties of these formulas can be found in Section 6.

Within the spherical shell $\rho_{\text {cen }} \leq \rho \leq \rho_{\text {rem }}$ neither the central nor the remote zonal harmonic series are convergent. Nevertheless, the source point can be arbitrarily chosen on the symmetry axis, and for the various source points we get several central and remote regions. Calculating the source constants for many source points, we can find a large spatial region where either the central or the remote zonal harmonic expansion formulas, for some source point, can be used to calculate the electric potential and field. Of course, close to the electrodes and dielectrics the zonal harmonic expansion method is not applicable (due to its divergence or very slow convergence); in these regions the electric potential and field of the axisymmetric system should be computed by elliptic integrals.

\section{SOURCE CONSTANTS FOR A CHARGED RING}

In order to calculate the electric field with the zonal harmonic expansion method, we need the source constant values. For a fixed source point, these numbers contain the whole information about the sources of the electric field (as far as the zonal harmonic expansion is concerned). We present in this section the calculation of the central and remote source constants for the simplest axisymmetric electric system: the circular charged ring.

We use the notations $Z, R$ and $Q$ for the axial coordinate, radius and charge of the ring, respectively, and we call the point $(Z, R)$ on the cylindrical meridian plane a charged ring point $C$. Let us fix on

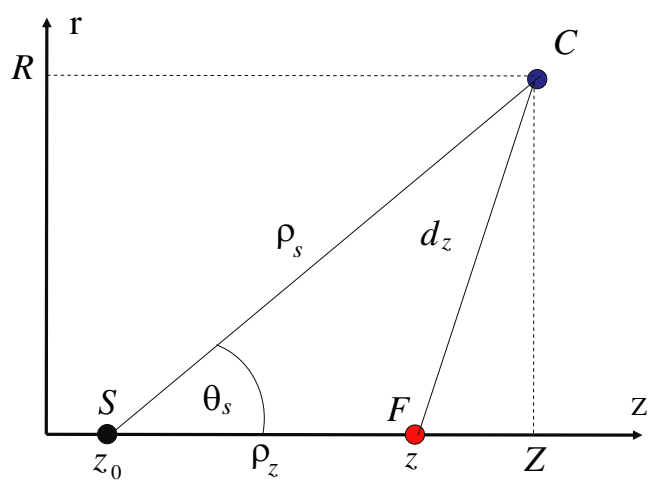

Figure 2. The charged ring point $C$, source point $S$, axial field point $F$ triangle. 
the symmetry axis $z$ a source point $S$ with axial coordinate $z_{0}$, and let us denote the distance between the source point $S$ and the ring point $C$ by $\rho_{s}$, and the angle between the symmetry axis $z$ and the $S-C$ line by $\theta_{s}$ (see Fig. 2). $\rho_{s}$ and $\cos \theta_{s}$ can be expressed as

$$
\rho_{s}=\sqrt{\left(Z-z_{0}\right)^{2}+R^{2}}, \quad u_{s}=\cos \theta_{s}=\left(Z-z_{0}\right) / \rho_{s} .
$$

Note that here the ring point $C$ and the source variables $Z, R, \rho_{s}$ and $u_{s}$ are analogous to the field point $F$ and to the field variables $z, r, \rho$ and $u$ of Section 2 .

Both the central and the remote convergence radii of the ring are equal to $\rho_{s}$. Nevertheless, we want to write here the source constant expressions in a general form, so that the charged ring could be later considered as part of a more complex electric system. Therefore, we assume below that $\rho_{c e n} \leq \rho_{s} \leq \rho_{\text {rem }}$, i.e., the ring is located between the sphere surfaces limiting the central and remote regions.

Let us now consider a special field point $F$ on the symmetry axis with axial coordinate $z\left(z>z_{0}\right)$. The electric potential at this point due to the charged ring is simply

$$
\Phi_{0}(z)=\frac{Q}{4 \pi \varepsilon_{0} d_{z}},
$$

where $d_{z}$ denotes the distance of the axial field point $F$ and the ring point $C$. The source point $S$, the axial field point $F$ and the ring point $C$ constitute a triangle with side lengths $d_{z}, \rho_{s}$ and $\rho_{z}=z-z_{0}$ (see Fig. 2). We can express the $d_{z}$ distance with the other parameters of this triangle:

$$
d_{z}=\sqrt{\rho_{s}^{2}+\rho_{z}^{2}-2 \rho_{s} \rho_{z} u_{s}} .
$$

Let us first assume that the axial field point $F$ is inside the central convergence region $\left(\rho_{z}<\rho_{c e n}\right)$; since $\rho_{\text {cen }} \leq \rho_{s}, \rho_{z}<\rho_{s}$ is then also valid. Introducing $h_{c e n}=\rho_{z} / \rho_{s}$, and using the generating function Formula (A1) of Appendix A, the $1 / d_{z}$ factor of Eq. (14) can be written in terms of a Legendre polynomial expansion as follows:

$$
\frac{1}{d_{z}}=\frac{1}{\rho_{s}} \sum_{n=0}^{\infty} h_{c e n}^{n} P_{n}\left(u_{s}\right) .
$$

Next, let us consider an axial field point $F$ inside the remote convergence region $\left(\rho_{z}>\rho_{\text {rem }}\right)$; since $\rho_{\text {rem }} \geq \rho_{s}, \rho_{z}>\rho_{s}$ is then also valid. In this case we define $h_{r e m}=\rho_{s} / \rho_{z}$. Recalling again Eq. (A1), we get now the following expansion:

$$
\frac{1}{d_{z}}=\frac{1}{\rho_{z}} \sum_{n=0}^{\infty} h_{r e m}^{n} P_{n}\left(u_{s}\right) .
$$


Inserting Eqs. (16) and (17) into Eq. (14), and comparing to Eqs. (2) and (9), where $u=P_{n}(u)=1$, we get the central and remote source constants of the charged ring:

$$
\begin{aligned}
\Phi_{n}^{c e n} & =\frac{Q}{4 \pi \varepsilon_{0} \rho_{s}}\left(\frac{\rho_{\text {cen }}}{\rho_{s}}\right)^{n} P_{n}\left(u_{s}\right), \\
\Phi_{n}^{r e m} & =\frac{Q}{4 \pi \varepsilon_{0} \rho_{s}}\left(\frac{\rho_{s}}{\rho_{\text {rem }}}\right)^{n+1} P_{n}\left(u_{s}\right) .
\end{aligned}
$$

If the electric system contains many charged rings, the source constants of the whole system can be obtained by summing these expressions over all rings.

If there are no other electrodes in addition to the ring, then $\rho_{\text {cen }}=\rho_{\text {rem }}=\rho_{s}$, so the central and remote source constants are equal, and their $n$-dependence follows the behavior of the Legendre polynomials, i.e., they decrease rather slowly with $n$ (see the asymptotic Formula (A7) in Appendix A). On the other hand, if the ring is considered as part of a larger electrode system, so that $\rho_{c e n}<\rho_{s}<\rho_{\text {rem }}$, then the source constant contributions of the ring decrease exponentially with $n$. These considerations are important to understand the convergence properties of the zonal harmonic expansions for a general axisymmetric electric system (for more details see Section 6).

\section{SOURCE CONSTANTS FOR AN AXISYMMETRIC ELECTRODE}

We present now results for the central and remote source constants for a general axially symmetric electrode. This could be, in principle, approximated by many charged rings, and in this case we could use the formulas of the previous section. Nevertheless, for practical problems we have usually electrodes with continuous charge density distributions, and then higher precision can be attained by integrations instead of summations.

In the case of a metallic electrode, the electric charge is present only on the electrode surface. The cross-section of the axisymmetric electrode with the $(z, r)$ meridian plane is a curve; let us assume that this curve is parametrized by the $Z(p), R(p)$ functions, where the parameter $p$ is the path length on the electrode curve. The charge density on the electrode surface is assumed to be $\sigma(p)$. The infinitesimal charge $d Q$ on the electrode surface part defined by the parameter interval $(p, p+d p)$ is

$$
d Q=2 \pi R(p) \sigma(p) d p .
$$


Replacing $Q$ in Eqs. (18) and (19) by $d Q$, and integrating over $p$, we get the central and remote source constants of the electrode:

$$
\begin{aligned}
\Phi_{n}^{c e n} & =\frac{1}{2 \varepsilon_{0}} \int d p \cdot \sigma \frac{R}{\rho_{s}}\left(\frac{\rho_{c e n}}{\rho_{s}}\right)^{n} P_{n}\left(u_{s}\right), \\
\Phi_{n}^{r e m} & =\frac{1}{2 \varepsilon_{0}} \int d p \cdot \sigma \frac{R}{\rho_{s}}\left(\frac{\rho_{s}}{\rho_{r e m}}\right)^{n+1} P_{n}\left(u_{s}\right) .
\end{aligned}
$$

In these formulas $\sigma, \rho_{s}$ and $u_{s}$ depend on the curve parameter $p$ (due to the $p$-dependence of $Z$ and $R$ in Eq. (13)). The central and remote convergence radii are:

$$
\rho_{\text {cen }}=\min _{p} \rho_{s}, \quad \rho_{\text {rem }}=\max _{p} \rho_{s} .
$$

The integrations can be performed by weighted sums of the integrand values at some number of discretization points (see Appendix C). Since the integrands in Eqs. (21) and (22) are computed by recurrence relations, one can save a lot of computation time by summing over the discretization points in an outer loop, and evaluating the integrands at fixed discretization points for all Legendre polynomial indices $n$ in an inner loop.

Usually, an electrode system contains many electrodes. The source constants of the whole system can be obtained by summing the source constant contributions from all electrodes. The central and remote convergence radii of the whole system are the minimal and maximal $\rho_{\text {cen }}$ and $\rho_{\text {rem }}$ values of Eq. (23), respectively, taken over all electrodes.

\section{SOURCE CONSTANTS FOR AN AXISYMMETRIC DIELECTRIC}

Let us denote the axial and radial coordinates of an arbitrary point of the dielectric by $Z$ and $R$. The axisymmetric dielectric has axial and radial polarization components: $\mathcal{P}_{z}=\mathcal{P}_{z}(Z, R), \mathcal{P}_{r}=\mathcal{P}_{r}(Z, R)$. In order to derive the source constants of the dielectric, we first calculate its on-axis scalar potential. Let us consider a small rectangle on the $(z, r)$ meridian plane with axial coordinates $Z$ and $Z+d Z$ and with radial coordinates $R$ and $R+d R$, where $d Z$ and $d R$ are infinitesimally small. This rectangle defines an electric dipole ring, with axial and radial dipole moments $p_{z}=\mathcal{P}_{z} d V$ and $p_{r}=\mathcal{P}_{r} d V$, where $d V=2 \pi R d Z d R$ is the volume of the ring. Using the dipole potential formula, we obtain the following on-axis scalar potential corresponding to this polarized ring:

$$
\Phi_{0}(z)=\frac{d V}{4 \pi \varepsilon_{0}}\left[\mathcal{P}_{z} \frac{z-Z}{d_{z}^{3}}-\mathcal{P}_{r} \frac{R}{d_{z}^{3}}\right],
$$


where $d_{z}$ denotes the distance of the on-axis point $(z, 0)$ from the ring.

To derive the central source constants corresponding to an arbitrary source point $z_{0}$, we assume first that $\rho_{z}=z-z_{0}<\rho_{s}$, where $\rho_{s}$ is the distance of the source point $z_{0}$ from the polarized ring (see Eq. (13)). Defining $h=\rho_{z} / \rho_{s}$, and writing $d_{z}=\rho_{s} \sqrt{1+h^{2}-2 h u_{s}}$ (see Eq. (15)), we can write the expression $\partial_{z} d_{z}^{-1}=-(z-Z) / d_{z}^{3}$ as a Legendre polynomial expansion, using Eq. (A1). Similarly, $d_{z}^{-3}$ can be expanded with the help of Eq. (A9). Comparing these expansions with the on-axis $\left(u=P_{n}(u)=1, \rho=\rho_{z}\right)$ version of Eq. (2), we get the central source constants for the polarized ring.

To get the remote source constants, we choose $\rho_{z}=z-z_{0}>\rho_{s}$, and we define $h=\rho_{s} / \rho_{z}$. Taking the above Legendre polynomial expansions and comparing with the on-axis version of Eq. (9), we get the remote source constants for the polarized ring.

Another (somewhat simpler) derivation is the following: first, the polarized ring with the above axial dipole moment $p_{z}$ can be substituted by 2 charged rings with axial coordinates $Z$ and $Z+d Z$, radii $R$, and charges $Q=p_{z} / d Z$ and $-Q$. Similarly, the radially polarized ring can be replaced by 2 charged rings with charges $Q=$ $p_{r} / d R$ and $-Q$, with the same axial coordinate $Z$, and with different radii $R$ and $R+d R$. Hence, the source constants of the axially and radially polarized rings can be expressed as axial and radial derivatives of the charged ring source constants:

$$
\begin{aligned}
& \Phi_{n}\left(\text { polarized ring, } p_{z}\right)=p_{z} \partial_{Z} \Phi_{n}(\text { charged ring, } Q=1), \\
& \Phi_{n}\left(\text { polarized ring, } p_{r}\right)=p_{r} \partial_{R} \Phi_{n}(\text { charged ring, } Q=1) .
\end{aligned}
$$

Using the charged ring source constants of Eqs. (18) and (19), and the derivative expressions of (B4), (B3), (B1) and (B2), we get the central and remote source constants of the polarized ring.

For a general axisymmetric dielectric, the central and remote source constants can be expressed by the following two-dimensional integrals:

$$
\begin{aligned}
\Phi_{n}^{c e n}= & -\frac{1}{2 \varepsilon_{0}} \int d R \int d Z \cdot \frac{R}{\rho_{s}^{2}}\left(\frac{\rho_{c e n}}{\rho_{s}}\right)^{n} \\
& \left\{(n+1) \mathcal{P}_{z} P_{n+1}\left(u_{s}\right)+\mathcal{P}_{r} \frac{R}{\rho_{s}} P_{n+1}^{\prime}\left(u_{s}\right)\right\} \\
\Phi_{n}^{r e m}= & \frac{1}{2 \varepsilon_{0}} \int d R \int d Z \cdot \frac{R}{\rho_{s}^{2}}\left(\frac{\rho_{s}}{\rho_{r e m}}\right)^{n+1}\left\{n \mathcal{P}_{z} P_{n-1}\left(u_{s}\right)-\mathcal{P}_{r} \frac{R}{\rho_{s}} P_{n-1}^{\prime}\left(u_{s}\right)\right\}
\end{aligned}
$$

(using the convention of Eq. (A3) for the $n=-1$ index). 
If the polarization functions $\mathcal{P}_{z}(Z, R)$ and $\mathcal{P}_{r}(Z, R)$ are independent of the axial variable $Z$, the integration over $Z$ can be carried out analytically:

$$
\begin{aligned}
\Phi_{n}^{c e n}= & \frac{1}{2 \varepsilon_{0} \rho_{c e n}} \int d R \cdot R\left[\left(\frac{\rho_{c e n}}{\rho_{s}}\right)^{n+1}\left\{\mathcal{P}_{z} P_{n}\left(u_{s}\right)+\mathcal{P}_{r} \frac{R}{n \rho_{s}} P_{n}^{\prime}\left(u_{s}\right)\right\}\right]_{Z_{\min }(R)}^{Z_{\max }(R)}, \\
\Phi_{n}^{r e m}= & \frac{1}{2 \varepsilon_{0} \rho_{r e m}} \int d R \cdot R\left[( \frac { \rho _ { s } } { \rho _ { r e m } } ) ^ { n } \left\{\mathcal{P}_{z} P_{n}\left(u_{s}\right)\right.\right. \\
& \left.\left.-\mathcal{P}_{r} \frac{R}{(n+1) \rho_{s}} P_{n}^{\prime}\left(u_{s}\right)\right\}\right]_{Z_{\min }(R)}^{Z_{\max }(R)},
\end{aligned}
$$

where we use the general notation $[f]_{a}^{b}=f(b)-f(a)$.

Equation (29) is singular for $n=0$. In this case we should use the integrated formula

$$
\Phi_{0}^{c e n}=\frac{1}{2 \varepsilon_{0}} \int d R \cdot\left[\mathcal{P}_{z} \frac{R}{\rho_{s}}-\mathcal{P}_{r} u_{s}\right]_{Z_{\min }(R)}^{Z_{\max }(R)} .
$$

Note that in the case of the integrated formula (29) the effective central convergence radius is the minimal distance of the source point from the axial boundary points $\left(Z_{\min }(R), R\right)$ and $\left(Z_{\max }(R), R\right)$. This is usually larger than the minimal distance of the source point from the dielectric. Detailed discussion about this convergence issue can be found in Section 6.

We have tested the above formulas by computing the potential and field of various axisymmetric dielectrics with 3-dimensional numerical integration of the dipole formulas, and comparing the results with the zonal harmonic expansions presented in Section 2, where the above expressions for the source constants have been used.

The polarization distribution $\mathcal{P}$ is equivalent to the sum of volume charge density distribution $\rho_{\mathrm{vol}}=-\nabla \cdot \mathcal{P}$ and surface charge density distribution $\sigma_{\text {surf }}=\mathcal{P} \cdot \mathbf{n}$, where $\mathbf{n}$ is the outwardly directed normal vector of the dielectric surface (see Refs. [41,42]). Using Eqs. (18), (19), (B3), (B4), (B1), (B2), the axisymmetric divergence formula $\nabla \cdot \mathcal{P}=\partial_{Z} \mathcal{P}_{z}+1 / R \cdot \partial_{R}\left(R \mathcal{P}_{r}\right)$, and integration by parts, we have checked that our source constant formulas indeed satisfy these equivalence relations.

We mention that the electric field of a dielectric can also be calculated by fictitious, equivalent magnetic currents [43]. 


\section{CONVERGENCE OF THE ZONAL HARMONIC EXPANSION}

\subsection{Convergence Radius and the Cauchy-Hadamard Theorem}

According to the Cauchy-Hadamard theorem [44,45], the radius of convergence of the power series

$$
f(x)=\sum_{n=0}^{\infty} c_{n} x^{n}
$$

is

$$
r_{c}=\left(\limsup _{n \rightarrow \infty}\left(\left|c_{n}\right|\right)^{1 / n}\right)^{-1}=\left(\lim _{n \rightarrow \infty}\left[\max _{m \leq n}\left(\left|c_{m}\right|\right)^{1 / m}\right]\right)^{-1} .
$$

The power series is convergent for $|x|<r_{c}$ and divergent for $|x|>r_{c}$.

The Cauchy-Hadamard theorem is very useful to get the convergence radii of the zonal harmonic expansion formulas. Let us start with the simplest electrode system consisting only of 1 charged ring. Eqs. (A7) and (A8) of Appendix A show that for $|u|<1$ the asymptotic $n$-dependence of the Legendre polynomial of order- $n$ and its first derivative is $P_{n} \sim 1 / \sqrt{n}$ and $P_{n}^{\prime} \sim \sqrt{n}$, respectively (for $|u|=1$ it is $\left|P_{n}\right|=1$ and $\left.P_{n}^{\prime} \sim n^{2}\right)$. Let us now write the zonal harmonic expansion Formulas (2)-(4), (9)-(11) into the form of Eq. (32), with $x=\mathcal{R}_{c}=\rho / \rho_{\text {cen }}$ (central expansions) or $x=\mathcal{R}_{c}=\rho_{\text {rem }} / \rho$ (remote expansions), respectively. Using the above asymptotic $n$-dependence formulas of the Legendre polynomials, and Eqs. (18) and (19), one can see that in the case of a charged ring the coefficients $c_{n}$ have a polynomial $n$-dependence of $c_{n} \sim n^{p}$, where $p$ is some small (positive or negative) number. Since $\lim _{n \rightarrow \infty} n^{p / n}=1$, it follows from Eq. (33) that for all zonal harmonic potential and field formulas $r_{c}=1$, i.e., the radius of convergence of the zonal harmonic expansions for the charged ring is the distance of the ring and the source point (this is true for both the central and the remote expansions).

In the case of many charged rings, $\rho_{\text {cen }}$ and $\rho_{\text {rem }}$ are defined as the minimal and maximal distance of the source point from the rings, respectively. From Eq. (18) it is obvious that for the central expansion the ring closest to the source point is dominant for large $n$, the source constant contributions of the rings farther away from the source point decrease rapidly with $n$. In the case of the remote expansion the ring that is farthest from the source point is dominant. A general axisymmetric electric system could be imagined as a collection of infinitely many charged rings. Therefore, it seems that we have 
generally proven: the radius of convergence of the central expansions of Eqs. (2)-(4) is the minimal distance of the source point from the electrodes and dielectrics $\left(\rho_{c e n}\right)$, and the radius of convergence of the remote expansions of Eqs. (9)-(11) is the maximal distance of the source point from the electrodes and dielectrics $\left(\rho_{\text {rem }}\right)$.

\subsection{Minimal and Effective Convergence Radii}

In the case of the central expansions the situation is, however, not so simple. Let us consider a charged cylinder with constant charge density, and let us approximate it with many charged rings. We compute then the source constants of the cylinder for a source point in the middle of the cylinder. From Eq. (18), we would naively think that the central source coefficients are in this case slowly decreasing functions of $n$. In reality, they decrease rapidly with $n$, like

$$
c_{n} \sim \lambda^{n} \quad(\lambda<1)
$$

For large $n$ the Legendre polynomials have a sinusoidal behavior (see Eq. (A7)), and it turns out that for the charged rings close to the minimal ring (which is closest to the source point) there occur large cancellations among the various ring contributions. The rapid (exponential) decrease of the central source constants with $n$ is the consequence of these cancellations. Analytical calculations for the charged cylinder with constant charge density show that the central source constant coefficients decrease exponentially with $n$, like in Eq. (34). One should emphasize here that numerical calculations of the central source constants (either with summation over the rings, or with numerical integration) are for large $n$ rather sensitive to numerical inaccuracies: loss of digits due to the cancellations (the 'small difference of large numbers' problem) stop the exponential decrease of the numerically computed source constants above some large $n$.

If the central source coefficients $c_{n}$ have the exponential decrease behavior of Eq. (34), the radius of convergence of Eq. (32) is not 1, but $r_{c}=1 / \lambda$, and the effective radius of convergence of the central zonal harmonic expansions of Eqs. (2)-(4) is not $\rho_{c e n}^{\min }$, i.e., the minimal distance of the source point from the electric system, but larger:

$$
\rho_{\text {cen }}^{\text {eff }}=\rho_{\text {cen }}^{\min } / \lambda \text {. }
$$

In the case of a cylinder with constant charge density, the effective convergence radius for a source point inside the cylinder is the minimal distance of the source point from the cylinder end corners (the $\left(Z_{\min }, R\right)$ and $\left(Z_{\max }, R\right)$ points; $\rho_{c e n}^{\min }=R$; see Fig. 3$)$. In the case of a disc with constant charge density, with axial coordinate $Z$, and 

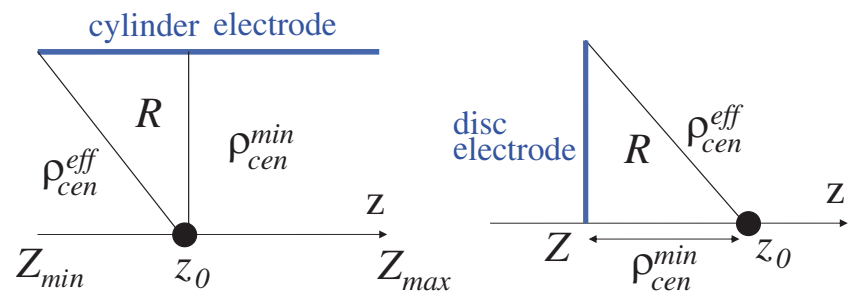

Figure 3. Minimal and effective central convergence radii for cylinder and disc electrodes with constant charge density.

with outer radius $R_{\text {out }}=R$ and inner radius $R_{\text {in }}=0$, the effective convergence radius is the distance between the source point and the outer disc point $(Z, R)\left(\rho_{\text {cen }}^{\text {eff }}=\sqrt{R^{2}+\left(z_{0}-Z\right)^{2}}\right.$, while $\rho_{c e n}^{\min }=\left|z_{0}-Z\right|$; see Fig. 3).

One can easily understand the above effective convergence radius of the finite cylinder electrode by replacing it as a superposition of an infinitely long cylinder electrode having constant charge density $\sigma$ in the whole axial region $(-\infty,+\infty)$ (this electrode has zero field inside, by symmetry argument), and of 2 infinitely long cylinder electrodes with charge density $-\sigma$ and with axial regions $\left(-\infty, Z_{\min }\right)$ and $\left(Z_{\max },+\infty\right)$, respectively. The central convergence radii of the latter 2 cylinders are obviously the minimal distances of the source point from the end points $\left(Z_{\min }, R\right)$ and $\left(Z_{\max }, R\right)$, respectively. Similarly, the charged disc can be replaced by a superposition of an infinitely large charged plane with constant charge density $\sigma$ (the electric field of this plane is simply $E_{z}= \pm \sigma /\left(2 \varepsilon_{0}\right)$ at the two sides), and of a hollow plane with constant charge density $-\sigma$ and with a disc hole of radius $R$. The central convergence radius of the hollow plane is the same as the effective convergence radius of the disc.

\subsection{Rate of Convergence}

Table 1 illustrates the rate of convergence with a few simple examples. One can find there the number of central zonal harmonic expansion terms that are necessary to obtain single $\left(10^{-7}\right)$ and double $\left(10^{-14}\right)$ precision for the potential of a charged ring, disc and cylinder (with constant charge densities), for various convergence ratios $\mathcal{R}_{c}=\rho / \rho_{c e n}$ and source point coordinates $z_{0}$. In order to assess the accuracy of the potential obtained from zonal harmonic series with some finite number of terms, we have compared the zonal harmonic potential result with the potential value computed by elliptic integrals, which have double precision accuracy in our codes. In the case of the charged ring, the convergence rate is similar to that of the simple geometric series. We 
Table 1. Number of central zonal harmonic terms $N_{\text {single }}$ and $N_{\text {double }}$ that are necessary to obtain single $\left(10^{-7}\right)$ and double $\left(10^{-14}\right)$ precision for the potential of a charged ring, disc and cylinder (the latter two having constant charge density), for various convergence ratios $\mathcal{R}_{c}=\rho / \rho_{\text {cen }}$ and source point coordinates $z_{0}$. Ring: $Z=0, R=1$. Cylinder: $Z_{\min }=-2, Z_{\max }=2, R=1$. Disc1: $Z=0, R_{\text {in }}=0$, $R_{\text {out }}=1$. Disc2: $Z=0, R_{\text {in }}=0.5, R_{\text {out }}=1$. Field point direction in all cases: $u=\cos \theta=0$.

\begin{tabular}{|c|l|c|c|c|c|c|c|}
\hline$R_{c}$ & 0.1 & 0.5 & 0.7 & 0.9 & 0.95 & 0.98 & 0.99 \\
\hline \hline Ring, $z_{0}=0.5: N_{\text {single }}$ & 6 & 18 & 32 & 106 & 202 & 478 & 898 \\
\hline Ring, $z_{0}=0.5: N_{\text {double }}$ & 12 & 40 & 74 & 248 & 500 & 1238 & 2402 \\
\hline \hline Cylinder, $z_{0}=5: N_{\text {single }}$ & 4 & 14 & 24 & 64 & 122 & 240 & 394 \\
\hline Cylinder, $z_{0}=5: N_{\text {double }}$ & 12 & 34 & 64 & 202 & 394 & 932 & 1722 \\
\hline \hline Cylinder, $z_{0}=0: N_{\text {single }}$ & 4 & 6 & 8 & 10 & 10 & 10 & 10 \\
\hline Cylinder, $z_{0}=0: N_{\text {double }}$ & 8 & 16 & 20 & 24 & 28 & 28 & 28 \\
\hline \hline Disc1, $z_{0}=1: N_{\text {single }}$ & 4 & 10 & 16 & 20 & 24 & 24 & 28 \\
\hline Disc1, $z_{0}=1: N_{\text {double }}$ & 10 & 24 & 36 & 52 & 60 & 64 & 68 \\
\hline \hline Disc2, $z_{0}=1: N_{\text {single }}$ & 4 & 14 & 26 & 68 & 122 & 250 & 406 \\
\hline Disc2, $z_{0}=1: N_{\text {double }}$ & 10 & 34 & 66 & 202 & 400 & 928 & 1774 \\
\hline \hline
\end{tabular}

can understand this fact from Eq. (18): the central source constants are then equal to some values of the Legendre polynomials, so they decrease rather slowly with $n$. In the case of the disc and cylinder electrodes with constant charge density, the convergence is faster than for the ring. This is due to the more rapid decrease of the source constants with $n$. If the source point is far from the cylinder $\left(z_{0}=5\right)$, the central source constants near $n=1000$ are about $10^{3}-10^{4}$ times smaller than at small $n$. This decrease of the source constants can be understood by the following consideration. Let us approximate the cylinder by many charged rings; then, it is obvious from Eq. (18) that for large $n$ the rings far from the source point contribute negligibly to the source constants, so only a small part of the cylinder electrode provides an essential contribution to the source constants. The size of this part decreases with $n$, therefore also the source constants themselves decrease with $n$. If the source point is inside the cylinder, the central source constants decrease extremely rapidly with $n$; in the case of the cylinder and $z=0$ source point of Table 1 the central source constants near $n=45$ are 18 orders of magnitude smaller than at small $n$. Theoretically, they would further decrease with $n$, but due to numerical inaccuracies of the double precision computation the decrease of the source constants 
stops at this level. As we have mentioned above, the fast decrease of the source constants in this case is due to cancellation effects among the sinusoidally oscillating Legendre polynomial terms. Similarly fast decrease of the central source constants happens in the case of disc1 (with $R_{\text {in }}=0$ ), therefore the zonal harmonic expansion convergence is very fast. However, in the case of $\operatorname{disc} 2$ (with $R_{i n}=0.5$ ) the above mentioned cancellation does not take place, so the source constants decrease only slowly, and the convergence is also much slower, as one can see from the much larger $N_{\text {single }}$ and $N_{\text {double }}$ numbers.

Cylinders and disks with constant charge density can be applied for electric field calculations of some special dielectrics (for example, a dielectric cylinder with constant axial polarization). One can use them also for practically interesting magnetic field calculations, if one changes the electric charges to equivalent (fictitious) magnetic charges. In addition, it turns out that the central source constants for source points inside cylindrical or conical electrodes with constant potential (i.e., not with constant charge density) decrease also rapidly with increasing $n$; this decrease is also a consequence of cancellation effects of Legendre polynomial terms with sinusoidal behavior.

\subsection{Connection with Complex Functions}

The zonal harmonic expansion of the electric potential (field) is convergent within a sphere which does not contain any charges, i.e., singular sources of the potential. This seems to be similar to the convergence and analyticity properties of complex power series. In fact, there exists indeed a close connection between the convergence properties of 3-dimensional axisymmetric real harmonic functions and complex functions, as it was first shown by G. Szegö in Ref. [46]. If

$$
U(\rho, \theta)=\sum_{n=0}^{\infty} a_{n} \rho^{n} P_{n}(\cos \theta)
$$

is an axially symmetric potential function defined by a central zonal harmonic expansion, with $\rho_{c e n}=\left(\lim \sup _{n \rightarrow \infty}\left|a_{n}\right|^{1 / n}\right)^{-1}$, and

$$
f(v)=\sum_{n=0}^{\infty} a_{n} v^{n}
$$

is the Taylor expansion of a corresponding complex function of $v=$ $\rho e^{i \theta}$, then both series converge for $\rho=|v|<\rho_{\text {cen }}$ and diverge for $\rho=|v|>\rho_{\text {cen }}$. The first series defines a regular axially symmetric potential in the sphere $\rho<\rho_{\text {cen }}$ in three dimensions, and the second series defines a regular analytic function in the circle $|v|<\rho_{\text {cen }}$ of the 
complex plane. Furthermore, the 3-dimensional real boundary ring $\rho=\rho_{\text {cen }}, \theta=\theta_{0}$ is a regular (singular) ring of the potential function $U$ if and only if the complex boundary point $v_{0}=\rho_{c e n} e^{i \theta_{0}}$ is a regular (singular) point of the complex function $f$.

This theorem shows us an interesting connection between sources of axisymmetric electric potentials and singularities of complex functions: a central zonal harmonic expansion of the potential is convergent within a sphere that does not contain any charges (sources of the potential), and the corresponding power series of the complex function is convergent within a circle that does not contain any singularities of the function. We mention that the remote zonal harmonic expansion is analogous to the $\sum_{n=-\infty}^{-1} a_{n} v^{n}$ part of the Laurent series in complex analysis.

\section{THE ZONAL HARMONIC EXPANSION IN PRACTICE}

In order to use the zonal harmonic expansion for practical electric potential and field calculations, the first step is to compute the charge density distribution on the surface of the electrodes and dielectrics, and the volume charge density in the dielectrics. The most natural method for this purpose is BEM, but in principle one could also use FDM or FEM (as we have discussed in Section 1).

The second step is the definition of the source points. They should be chosen in such a way that the central zonal harmonic expansion is convergent within a large region inside the electric system. The optimal distance between two neighboring central source points should be a few times smaller than the central convergence radius at these points; otherwise, it could happen that the central zonal method is not convergent at some points near the axis. For many electric field computation applications it is not necessary to define remote source points, as the central source points are sufficient for the field calculations inside the electric system.

The next step is the calculation of the source constants for all source points. In the beginning, the user (or the code) has to decide on the maximal source constant index $n_{\max }$ for each source point. The optimal choice of $n_{\max }$ depends on the maximal value of the convergence ratio that is expected to be used during the field calculation; typically, this is dependent on whether one intends to calculate the field close to the electrodes (dielectrics) or not. If the maximal convergence ratio is expected to be not too close to 1 (for example: 0.9 ), a relatively small value for $n_{\max }$ can be chosen (like 250; compare with Table 1). On the other hand, in regions where accurate 
field computations close to electrodes or dielectrics are necessary or, more generally, where the convergence ratio is expected to be very close to 1 (e.g., 0.98 or 0.99 ), a large $n_{\text {max }}$ value has to be defined (like 1000). The typical computation time of the central source constants, with a few hundred source points and with $n_{\max }=500$, is an order of minute (with our notebook, which has about $0.5 \mathrm{~ns}$ multiplication time). In addition to the source constants, also the convergence radii for all source points have to be computed. At the end, the source points, convergence radii and source constants should be saved to the hard disk, so that they could be used for a field computation later.

In the beginning of a field calculation, the source points, convergence radii and source constants have to be read from the hard disk into the main memory. In order to compute the electric potential and field at an arbitrary field point, the computer program first has to search for the best central source point, i.e. that source point for which the convergence ratio $\mathcal{R}_{c}=\rho / \rho_{\text {cen }}$ is minimal. In the beginning of a trajectory calculation, the program should search among all the source points, in order to find the best one. Later, however, it is enough to search for source points only close to the best source point of the last trajectory step, because the particle usually travels only a small distance during one step. If the central zonal harmonic expansion is not convergent for the best source point $\left(\rho / \rho_{\text {cen }}>1\right)$, or the convergence is too slow (e.g., $\rho / \rho_{\text {cen }}>0.98$ ), the elliptic integral or some other method has to be used for the field calculation.

An important practical question is the truncation criterion for the zonal harmonic series: at which index $n$ should one stop the expansion of Eqs. (2)-(4) and (9)-(11), in order to get some prescribed accuracy? In our codes, to get double precision accuracy for the potential and the 2 field components, we use the following procedure: the potential and field components are computed together with the same expansion, and the expansion is stopped if the absolute values of the last two terms for the potential and both field components are $10^{15}$ times smaller than the sum of the corresponding series. A similar (but slightly different) truncation criterion was suggested by Garrett in $[18,19]$.

The region where the fast zonal harmonic expansion cannot be used is usually small, therefore it might happen that the slow elliptic integral computation close to the electrodes is acceptable for the user. If this is not the case, the user has several possibilities to increase the computation speed. First, close to the electrodes one could compute a field map (field values at many grid points), and to use some kind of interpolation method to calculate the field values in between the grid points. The field map calculation is in this case rather slow; nevertheless, the field calculation with interpolation is 
fast, and this could then be sufficient to perform a fast trajectory calculation. Another possibility is to divide the electric system into several smaller groups, so that one could use the central or remote zonal harmonic expansion and the elliptic integral calculation for each group separately. With an optimal grouping, one could reduce the field computation time, by decreasing the size of the elliptic integral regions (where the zonal harmonic expansion is not convergent), or by decreasing the number of electrodes, dielectrics or electric subelements that have to be computed with the elliptic integral method.

At the end of this section, we compare the computational speed of the zonal harmonic method with the elliptic integral calculation in the case of a practically interesting problem. With our notebook (multiplication time: $0.5 \mathrm{~ns}$ ) we have made a computation for the electric potential of the KATRIN main spectrometer [10]. The charge density calculation with BEM and with a discretization of 1800 subelements took about 20 seconds, the central source constant computation time with 600 source points and with $n_{\max }=500$ was 40 seconds. Then, using the elliptic integrals (summing over all subelements), the computation time for the potential at a point near the middle of the spectrometer was $7 \mathrm{~ms}$. With the zonal harmonic expansion method, the computation time values for the potential and field components at points with convergence ratios of $0.5,0.8$ and 0.9 were $2 \mu \mathrm{s}, 6 \mu \mathrm{s}$ and $14 \mu \mathrm{s}$, respectively. This example illustrates that for electric field and potential calculations the zonal harmonic expansion method is by several orders of magnitude faster than the elliptic integral method.

\section{CONCLUSIONS}

We have presented the central and remote zonal harmonic expansion method for electric field calculations of axially symmetric electrodes and dielectrics. The zonal harmonic field series formulas are convergent at field points within the central and remote regions, which have spherical boundaries, and their center, the source point, can be arbitrarily chosen on the symmetry axis. The rate of convergence of the field series depends on the distance of the field and the source point; smaller distance for central field points and larger distance for remote field points correspond to higher convergence rate. For a given field point, one can improve the convergence properties of the zonal harmonic method by optimal choice of the field expansion method (central or remote) and of the source point. In order to use the zonal harmonic formulas for field calculations, one needs the source constants, which depend on the source point and on the 
geometrical and source strength properties of the electric system. We have presented source constant computation formulas for charged rings and for general axisymmetric electrodes and dielectrics.

The zonal harmonic electric field calculation method has several important advantages. First, the field and source equations are separated: during the source constant computations, one has to use only the source point and source parameters (geometry, potentials, permittivity), but not the field point parameters, and during the field computation, the source constants contain already the whole information about the electric sources. As an important consequence, electric field calculation with the zonal harmonic method is much faster (in some cases even 1000 times) than the widely known elliptic integral method. Second, the zonal harmonic method has not only high speed, but also high accuracy, which makes the method especially appropriate for trajectory calculations of charged particles. Due to these properties, no interpolation is necessary when the electric field during particle trajectories is computed with the aid of the zonal harmonic method. Third, the zonal harmonic method is more general and for practical applications more advantageous than the radial series expansion method, which is more widely known in the electron optics literature than the zonal harmonic method. In addition, the zonal harmonic field series formulas are relatively easy to differentiate and integrate, in contrast to the elliptic integral formulas.

The axisymmetric zonal harmonic method could be generalized to the spherical harmonic method, for electric field calculation of general three-dimensional systems. In that case, we have two-dimensional spherical harmonic expansions, instead of the one dimensional zonal harmonic expansions. The source point can then be an arbitrary point in space (not restricted to any symmetry axis), and the central and remote convergence radii are, similarly to the zonal harmonic method, the minimal and maximal distances between the source point and the electric sources (electrodes and dielectrics), respectively. Due to the two-dimensionality of the series, this three-dimensional method is probably fast enough only for convergence ratios that are much smaller than 1.

\section{ACKNOWLEDGMENT}

I would like to thank Profs. W. Heil, E. Otten and G. Drexlin for the possibility of long stays at the University of Mainz and at KIT (former University of Karlsruhe and Forschungszentrum Karlsruhe), supported by the German Federal Ministry of Education and Research (BMBF) under Contract Nos. 05CK1UM1/5, 05A08VK2 and 05CK5VKA/5. 
We acknowledge support by the Deutsche Forschungsgemeinschaft and the Open Access Publishing Fund of Karlsruhe Institute of Technology. Thanks to K. Valerius and T. J. Corona for many useful discussions.

\section{APPENDIX A. LEGENDRE POLYNOMIALS}

The Legendre polynomials $P_{n}(u)$ of order $n$ can be defined by the following generating function:

$$
\frac{1}{\sqrt{1+h^{2}-2 h u}}=\sum_{n=0}^{\infty} h^{n} P_{n}(u),
$$

where $|h|<1,|u| \leq 1$ (see Refs. [16,47-51]). These are the first 5 Legendre polynomials:

$$
\begin{aligned}
& P_{0}(u)=1, \quad P_{1}(u)=u, \quad P_{2}(u)=\left(3 u^{2}-1\right) / 2, \\
& P_{3}(u)=\left(5 u^{3}-3 u\right) / 2, \quad P_{4}(u)=\left(35 u^{4}-30 u^{2}+3\right) / 8 .
\end{aligned}
$$

In some Legendre polynomial formulas also the $n=-1$ index can occur. In order that the formulas are valid for this index, one has to use the convention

$$
P_{-1}(u)=P_{-1}^{\prime}(u)=P_{-1}^{\prime \prime}(u)=0,
$$

where $P_{n}^{\prime}=P_{n}^{\prime}(u)$ and $P_{n}^{\prime \prime}=P_{n}^{\prime \prime}(u)$ denote the first and second derivatives of the Legendre polynomial $P_{n}=P_{n}(u)$.

Special values of the Legendre polynomials are the following:

$$
\begin{aligned}
P_{n}(1) & =1, \quad P_{n}(-1)=(-1)^{n}, \\
P_{2 n+1}(0) & =0, \quad P_{2 n}(0)=(-1)^{n} \frac{(2 n) !}{\left(2^{n} n !\right)^{2}}, \\
P_{n}^{\prime}( \pm 1) & =( \pm 1)^{n+1} n(n+1) / 2 .
\end{aligned}
$$

Asymptotic formulas for large $n$ :

$$
\begin{aligned}
& P_{n}(u) \approx \sqrt{\frac{2}{\pi n \sin \theta}} \sin [(n+1 / 2) \theta+\pi / 4], \\
& P_{n}^{\prime}(u) \approx-\sqrt{\frac{2 n}{\pi \sin ^{3} \theta}} \sin [(n+1 / 2) \theta+3 \pi / 4],
\end{aligned}
$$

with $u=\cos \theta$.

Differentiating both sides of Eq. (A1) over $u$ we obtain

$$
\frac{1}{\left(1+h^{2}-2 h u\right)^{3 / 2}}=\sum_{n=0}^{\infty} h^{n} P_{n+1}^{\prime}(u) .
$$


As one can easily see from Eqs. (A1) and (A9), the Legendre polynomials and their first derivatives have the following symmetry properties:

$$
P_{n}(-u)=(-1)^{n} P_{n}(u), \quad P_{n}^{\prime}(-u)=(-1)^{n+1} P_{n}^{\prime}(u) .
$$

Recurrence relations are extremely useful for the analytical and numerical investigations connected with Legendre polynomials (as it was emphasized by Garrett in Ref. [17]). The following 2 recurrence relations are recommended for the fast computation of the Legendre polynomials and their first derivatives (for $n>1$ ):

$$
\begin{aligned}
& P_{n}=2 u P_{n-1}-P_{n-2}-\left(u P_{n-1}-P_{n-2}\right) / n, \\
& P_{n}^{\prime}=2 u P_{n-1}^{\prime}-P_{n-2}^{\prime}+\left(u P_{n-1}^{\prime}-P_{n-2}^{\prime}\right) /(n-1),
\end{aligned}
$$

with $P_{0}^{\prime}(u)=0, P_{1}^{\prime}(u)=1$.

The following recurrence relation is valid for arbitrary higher derivatives $P_{n}^{(m)}=d^{m} P_{n} / d u^{m}$ (for $\left.n>m\right)$ :

$$
P_{n}^{(m)}=2 u P_{n-1}^{(m)}-P_{n-2}^{(m)}+\frac{2 m-1}{n-m}\left(u P_{n-1}^{(m)}-P_{n-2}^{(m)}\right) .
$$

The starting derivatives for small $m$ can be calculated by Eq. (A2); for $m=2: P_{1}^{\prime \prime}=P_{1}^{(2)}=0, P_{2}^{\prime \prime}=P_{2}^{(2)}=3$.

In some special cases, one needs the Legendre polynomials with only even or only odd indices. Then it is expedient to use the following recurrence relations (taken from Ref. [17]):

$$
\begin{aligned}
P_{n} & =\left[\left(A u^{2}-B\right) P_{n-2}-C P_{n-4}\right] / M, \\
P_{n}^{\prime} & =\left[\left(A^{\prime} u^{2}-B^{\prime}\right) P_{n-2}^{\prime}-C^{\prime} P_{n-4}^{\prime}\right] / M^{\prime}, \\
M & =(n-1) n(2 n-5), M^{\prime}=(n-2)(n-1)(2 n-5), \\
A & =(2 n-5)(2 n-3)(2 n-1), A^{\prime}=A, \\
B & =2(n-2)^{2}(2 n-1)-1, B^{\prime}=2(n-2) n(2 n-5)-3, \\
C & =(n-2)(n-3)(2 n-1), C^{\prime}=(n-2)(n-1)(2 n-1) .
\end{aligned}
$$

There are several other mixed recurrence relations that contain both $P_{n}$ and $P_{n}^{\prime}$ (see Ref. [17]):

$$
\begin{aligned}
n P_{n} & =u P_{n}^{\prime}-P_{n-1}^{\prime}, \\
(n+1) P_{n} & =P_{n+1}^{\prime}-u P_{n}^{\prime}, \\
(2 n+1) P_{n} & =P_{n+1}^{\prime}-P_{n-1}^{\prime}, \\
\left(1-u^{2}\right) P_{n}^{\prime} & =n\left(P_{n-1}-u P_{n}\right), \\
\left(1-u^{2}\right) P_{n}^{\prime} & =(n+1)\left(u P_{n}-P_{n+1}\right) .
\end{aligned}
$$


By differentiating the above equations over $u$, we obtain useful relations containing the second derivatives $P_{n}^{\prime \prime}$ :

$$
\begin{aligned}
& \left(1-u^{2}\right) P_{n}^{\prime \prime}=(n+2) u P_{n}^{\prime}-n P_{n+1}^{\prime}, \\
& \left(1-u^{2}\right) P_{n}^{\prime \prime}=(n+1) P_{n-1}^{\prime}-(n-1) u P_{n}^{\prime}, \\
& \left(1-u^{2}\right) P_{n}^{\prime \prime}=2 P_{n-1}^{\prime}-n(n-1) P_{n}, \\
& \left(1-u^{2}\right) P_{n}^{\prime \prime}=2 P_{n+1}^{\prime}-(n+1)(n+2) P_{n} .
\end{aligned}
$$

Further details about the Legendre polynomials can be found in Refs. $[16,17,47-51]$.

\section{APPENDIX B. CYLINDRICAL DERIVATIVES OF SOLID ZONAL HARMONICS}

The central and remote solid zonal harmonic functions are defined by the expressions $\rho^{n} P_{n}(u)$ and $\rho^{-(n+1)} P_{n}(u)$, respectively $(n=0,1, \ldots)$. Here $\rho=\sqrt{\left(z-z_{0}\right)^{2}+r^{2}}$ denotes the distance between the source point $\left(z_{0}, 0\right)$ and the field point $(z, r)$, and $u=\cos \theta=\left(z-z_{0}\right) / \rho$ (cosine of the angle between the $z$ axis and the line going through the source and field points; see Fig. 1). We use also the notation $s=\sin \theta=\sqrt{1-u^{2}}=r / \rho$.

As stated in Section 2, the electric potential of an axially symmetric electric system in the source-free central convergence region $\left(\rho<\rho_{c e n}\right)$ can be generally expressed as an expansion of the central zonal harmonic functions $\rho^{n} P_{n}(u)$. Similarly, in the source-free remote convergence region $\left(\rho>\rho_{\text {rem }}\right)$ the potential can be written as an expansion of the remote zonal harmonic functions $\rho^{-(n+1)} P_{n}(u)$ (since both kinds of functions satisfy Laplace's equation).

In order to calculate the cylindrical components of the electric field, we need the derivatives of the zonal harmonic functions over the cylindrical coordinates $z$ and $r$. The derivative expressions of the central zonal harmonics are the following (see Refs. [17-20]):

$$
\begin{aligned}
& \partial_{z}\left(\rho^{n} P_{n}\right)=n \rho^{n-1} P_{n-1}, \\
& \partial_{r}\left(\rho^{n} P_{n}\right)=-s \rho^{n-1} P_{n-1}^{\prime} .
\end{aligned}
$$

The derivatives of the remote zonal harmonics can be written as (see Ref. [52]):

$$
\begin{aligned}
& \partial_{z}\left(\rho^{-(n+1)} P_{n}\right)=-(n+1) \rho^{-(n+2)} P_{n+1}, \\
& \partial_{r}\left(\rho^{-(n+1)} P_{n}\right)=-s \rho^{-(n+2)} P_{n+1}^{\prime} .
\end{aligned}
$$


Further useful derivatives of functions similar to the zonal harmonics are the following:

$$
\begin{aligned}
\partial_{z}\left(\rho^{n-1} P_{n}^{\prime}\right) & =(n+1) \rho^{n-2} P_{n-1}^{\prime}, \\
\partial_{r}\left(\rho^{n-1} P_{n}^{\prime}\right) & =-s \rho^{n-2} P_{n-1}^{\prime \prime}, \\
\partial_{z}\left(\rho^{-(n+2)} P_{n}^{\prime}\right) & =-n \rho^{-(n+3)} P_{n+1}^{\prime}, \\
\partial_{r}\left(\rho^{-(n+2)} P_{n}^{\prime}\right) & =-s \rho^{-(n+3)} P_{n+1}^{\prime \prime} .
\end{aligned}
$$

Using these relations and writing the $\Delta$ operator in cylindrical coordinates, we can check that the zonal harmonics really satisfy the Laplace's equation: $\Delta\left(\rho^{n} P_{n}\right)=0, \Delta\left(\rho^{-(n+1)} P_{n}\right)=0$.

The above derivative formulas can be proven by using the cylindrical derivative equations $\partial_{z} \rho=u, \partial_{z} u=\left(1-u^{2}\right) / \rho=s^{2} / \rho$, $\partial_{r} \rho=s=r / \rho, \partial_{r} u=-s u / \rho$, and the recurrence relations of the Legendre polynomials, presented in Appendix A. For example,

$$
\partial_{z}\left(\rho^{n} P_{n}\right)=n \rho^{n-1} u P_{n}+\rho^{n} P_{n}^{\prime}\left(1-u^{2}\right) / \rho=\rho^{n-1}\left[n u P_{n}+\left(1-u^{2}\right) P_{n}^{\prime}\right],
$$

and using the recurrence relation of Eq. (A23) we get Eq. (B1).

\section{APPENDIX C. NUMERICAL INTEGRATION}

We present here a simple one-dimensional numerical integration formula, based on 10th order equidistant Lagrange interpolation. The definite integral of the function $f(x)$ over the interval $[a, b]$ can be

Table C1. The numerical integration weight factors $w_{0}$ through $w_{9}$.

\begin{tabular}{|c|c|}
\hline$i$ & $w_{i}$ \\
\hline \hline 0 & 0.2803440531305107 \\
\hline 1 & 1.648702325837748 \\
\hline 2 & -0.2027449845679092 \\
\hline 3 & 2.797927414021179 \\
\hline 4 & -0.9761199294532843 \\
\hline 5 & 2.556499393738999 \\
\hline 6 & 0.1451083002645404 \\
\hline 7 & 1.311227127425048 \\
\hline 8 & 0.9324249063051143 \\
\hline 9 & 1.006631393298060 \\
\hline \hline
\end{tabular}


approximated by the following weighted sum of the function values:

$$
\int_{a}^{b} d x \cdot f(x) \approx \delta \sum_{i=0}^{N} w_{i} \cdot f(a+i \delta),
$$

where the number of discretization points is $N+1$ (with $N \geq 20$ ), $\delta=(b-a) / N$ is the distance between neighboring points, and the weight factors $w_{0}, \ldots, w_{9}$ are given in Table C1. The other weight factors $w_{10}, \ldots, w_{N}$ are the following:

$$
w_{i}=\left\{\begin{array}{l}
1: \text { for } 10 \leq i \leq N-10 \\
w_{N-i}: \text { for } i>N-10 .
\end{array}\right.
$$

In many cases (if the function has no sharp peaks or large higher derivatives in the integration interval), the above formula with $N=20$ provides close to double precision value for the integral. Otherwise, the integration error decreases with a high power of $1 / N$.

References [53,54] contain many other numerical integration algorithms.

\section{REFERENCES}

1. Paszkowski, B., Electron Optics, London Eliffe Books, American Elsevier, New York, 1968.

2. Szilágyi, M., Electron and Ion Optics, Plenum Press, New York and London, 1988.

3. Hawkes, P. W. and E. Kasper, Principles of Electron Optics, Vol. 1, Academic Press, Harcourt Brace Jovanovich, 1989.

4. Kraus, C., et al., "Final results from phase II of the Mainz neutrino mass search in tritium $\beta$ decay," Eur. Phys. J. C, Vol. 40, 447, 2005.

5. Lobashev, V. M., "The search for the neutrino mass by direct method in the tritium beta-decay and perspectives of study it in the project KATRIN," Nucl. Phys. A, Vol. 719, 153c, 2003.

6. Glück, F., et al., "The neutron decay retardation spectrometer aSPECT: Electromagnetic design and systematic effects," Eur. Phys. J. A, Vol. 23, 135, 2005.

7. Baessler, S., et al., "First measurements with the neutron decay spectrometer aSPECT," Eur. Phys. J. A, Vol. 38, 17, 2008.

8. Beck, M., et al., "WITCH: A recoil spectrometer for weak interaction and nuclear physics studies," Nucl. Instrum. Methods A, Vol. 503, 567, 2003.

9. Friedag, P., "Bahnverfolgungssimulationen für das WITCHexperiment," Diploma thesis, University of Münster, 2008. 
10. Angrik, J., et al., "KATRIN design report 2004," FZKA Scientific Report 7090, Forschungszentrum Karlsruhe, 2005, http://bibliothek.fzk.de/zb/berichte/FZKA7090.pdf.

11. Chari, M. V. K. and S. J. Salon, Numerical Methods in Electromagnetism, Academic Press, San Diego, 2000.

12. Maxwell, J. C., A Treatise on Electricity and Magnetism, Vol. 1, Clarendon Press, 1873.

13. Durand, E., Electrostatique, Vol. 1, Masson et Cie, Paris, 1964.

14. Jackson, J. D., Classical Electrodynamics, John Wiley \& Sons, New York, 1999.

15. Smythe, W. R., Static and Dynamic Electricity, McGraw Hill Book Company, New York, 1968.

16. Kellogg, O. D., Foundations of Potential Theory, Springer Verlag, Berlin, 1967.

17. Garrett, M. W., "Axially symmetric systems for generating and measuring magnetic fields," J. Appl. Phys., Vol. 22, 1091-1107, 1951.

18. Garrett, M. W., "The method of zonal harmonics," High Magnetic Fields, H. Kolm, et al. (eds.), John Wiley and Sons, 1962.

19. Garrett, M. W., "Computer programs using zonal harmonics for magnetic properties of current systems with special reference to the IBM 7090," ORNL-3318, Oak Ridge National Laboratory, USA, 1962.

20. Garrett, M. W., "Thick cylindrical coil systems for strong magnetic fields with field or gradient homogeneities of the 6th to 20th order," J. Appl. Phys., Vol. 38, 2563, 1967.

21. Thümmler, T., "Präzisionsüberwachung und Kalibration der Hochspannung für das KATRIN-experiment," Dissertation, University of Münster, 2007.

22. Pocanic, D., et al., "Nab: Measurement principles, apparatus and uncertainties," Nucl. Instrum. Methods A, Vol. 611, 211, 2009.

23. Valerius, K., "Elektromagnetisches Design für das Hauptspektrometer des KATRIN Experiments," Diploma thesis, University of Bonn, 2004.

24. Valerius, K., "Spectrometer-related background processes and their suppression in the KATRIN experiment," Dissertation, University of Münster, 2009.

25. Hugenberg, K., "Design of the electrode system for the KATRIN main spectrometer," Diploma thesis, University of Münster, 2008. 
26. Zacher, M., "Electromagnetic design and field emission studies for the inner electrode system of the KATRIN main spectrometer," Diploma thesis, University of Münster, 2009.

27. Wandkowsky, N., "Design and background simulations for the KATRIN main spectrometer and air coil system," Diploma thesis, Karlsruhe Institute of Technology, 2009.

28. Fränkle, F., "Background investigations of the KATRIN prespectrometer," Dissertation, Karlsruhe Institute of Technology, 2010.

29. Groh, S., "Untersuchung von UV-laser induziertem Untergrund am KATRIN Vorspektrometer," Diploma thesis, Karlsruhe Institute of Technology, 2010.

30. Hein, J. H. C., "Angular defined photo-electron sources for the KATRIN experiment," Diploma thesis, University of Münster, 2010.

31. Valerius, K., et al., "Prototype of an angular-selective photoelectron calibration source for the KATRIN experiment," J. Inst., Vol. 6, P01002, 2011.

32. Lukic, S., et al., "Ion source for tests of ion behavior in the KATRIN beam line," Rev. Sci. Instrum., Vol. 82, 013303, 2011.

33. KATRIN homepage, Talks and Publications, Diploma and Ph.D. theses, http://www-ik.fzk.de/ katrin/publications/thesis.html.

34. Westfälische Wilhelms-Universität Münster, Institut für Kernphysik, AG Prof. Dr. C. Weinheimer, http://www.unimuenster.de/Physik.KP/AGWeinheimer/Arbeiten-de.html.

35. Vöcking, S., "Implementierung der multipole boundary element methode für das KATRIN-experiment," Diploma thesis, University of Münster, 2008.

36. Corona, T. J., "Tools for electromagnetic field simulation in the KATRIN experiment," master thesis, MIT, 2009.

37. Leiber, B., "Non-axially symmetric field and trajectory calculations for the KATRIN experiment," Diploma thesis, Karlsruhe Institute of Technology, 2010.

38. Babutzka, M., et al., "The comprehensive guide to KASSIOPEIA," version 1.00.00, KATRIN internal report.

39. Böttcher, C. J. F., Theory of Electric Polarization, Vol. 1, Elsevier Sci. Publ. Comp., Amsterdam, 1973.

40. Griffiths, D. J., Introduction to Electrodynamics, Prentice Hall, Upper Saddle River, New Jersey, 1999.

41. Cowan, E. W., Basic Electromagnetism, Academic Press, New York, 1968. 
42. Panofsky, W. K. H. and M. Phillips, Classical Electricity and Magnetism, Dover Publications, Mineola, New York, 1962.

43. Ravaud, R., G. Lemarquand, and S. I. Babic, "Introducing fictitious currents for calculating analytically the electric field in cylindrical capacitors," Progress In Electromagnetics Research M, Vol. 9, 139-150, 2009.

44. Gamelin, T. W., Complex Analysis, Springer-Verlag, New York, 2001.

45. Needham, T., Visual Complex Analysis, Clarendon Press, Oxford, 1997.

46. Szegö, G., "On the singularities of zonal harmonic expansions," J. Rat. Mech. Anal., Vol. 3, 561, 1954.

47. Sneddon, I. N., Special Functions of Mathematical Physics and Chemistry, Longman, London and New York, 1980.

48. Rainville, E. D., Special Functions, The MacMillan Company, New York, 1960.

49. Bell, W. W., Special Functions for Scientists and Engineers, D. Van Nostrand Company Ltd., London, 1965.

50. Andrews, L. C., Special Functions of Mathematics for Engineers, Oxford University Press, Oxford, 1998.

51. Boas, M. L., Mathematical Methods in the Physical Sciences, John Wiley \& Sons, New York, 2006.

52. Frerichs, V., W. G. Kaenders, and D. Meschede, "Analytic construction of magnetic multipoles from cylindric permanent magnets," Appl. Phys. A, Vol. 55, 242, 1992.

53. Evans, G., Practical Numerical Integration, John Wiley \& Sons, Chichester, 1993.

54. Kythe, P. K. and M. R. Schäferkotter, Handbook of Computational Methods for Integration, Chapman\& Hall/CRC, London, 2005. 